\title{
New Scatterometer for Spatial Distribution Measurements of Light Scattering from Materials
}

\author{
${ }^{1,3}$ E. Kawate, ${ }^{1,2}$ M. Hain \\ ${ }^{1}$ AIST, 1-1-1, Tsukuba Central 2, Umezono, Tsukuba, Ibaragi 305-8568, Japan \\ ${ }^{2}$ Institute of Measurement Science, Slovak Academy of Sciences, Bratislava, Slovakia \\ ${ }^{3}$ TRAS, Inc., 725-28 Shimohirooka, Tsukuba, Ibaragi 305-0042, Japan, e.kawate@aist.go.jp
}

\begin{abstract}
A new scatterometer is composed of two ellipsoidal mirrors of revolution and an optical detection system. It enables us to absolutely measure diffuse reflectance and transmittance and to measure the spatial distribution of light scattering from almost all materials. The optical detection system has been developed both to measure total photo-intensity using a photodiode and to capture the imaging data using a CCD camera. This results in faster, more complete and often more accurate measurements than can be achieved with traditional goniometric methods and integrated sphere methods. The absolute total integrated reflectance and transmittance of well-known samples were measured and the spatial distribution of light scattering from a diffraction grating was captured and evaluated.
\end{abstract}

Keywords: STAR GEM ${ }^{\circledR}$, CCD camera, fish-eye lens, absolute reflectance, bi-directional reflection distribution function, total integrated reflectance

\section{INTRODUCTION}

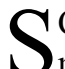
CATTERING MEASUREMENTS [1] provide us with material characterization based on scatter for metals, plastics, paper, textiles and also with surface treatment characterization based on scatter for cleaners, polishes, paints, and coatings. Not only TIR/TIT (Total Integrated Reflectance / Transmittance) [2] but also BRDF/BTDF (Bidirectional Reflection / Transmission Distribution Function) [3] is mainly measured in the scattering measurements.

Traditionally, BRDF/BTDF measurements are performed using goniometric systems [1] [3], which involve tilting and rotating a sample and/or a detector around the sample center in order to measure the BRDF/BTDF at selected points on the sphere around the sample, but these systems are relatively slow, potentially requiring hours to measure the sample. Nominally TIR/TIT measurements are made by integrating the BRDF/BTDF over a portion of the sphere around the sample, but actually they are measured using an integrating sphere [1] [2]. Although the measurement using the integrating sphere is fast, it is made only at a fixed angle of incidence and the spatial distribution measurement of light scattering from the sample cannot be made using the integrating sphere.

Recently new measurement methods based on a CCD camera have been developed. These methods try to resolve the flaw that the goniometric method requires long hours to measure the BRDF/BTDF. An Imaging Sphere ${ }^{\circledR}$ is an example of fast measurement of angular distribution of scattered light from surfaces. It consists of a hemisphere, a small convex mirror and a CCD camera. A sample is set in the center of the hemisphere. Incident light is introduced through a small hole in the hemisphere and irradiates the sample. Scattered light from the sample is diffusely reflected on the inner wall of the hemisphere. A fraction of the scattered light is reflected on the convex mirror, which is fixed near the sample, and the CCD camera captures the light from the convex mirror. The Imaging Sphere can acquire $2 \pi$ steradians of scattered light in a single shot and can dramatically reduce the time required to measure the BRDF/BTDF. There are several problems, (1) nonuniformity of the diffuse reflectance of the inner wall of the hemisphere, (2) inter-reflections between the CCD camera and the sample and (3) asymmetric position of the convex mirror.

Almost all devices for the measurements of reflectance and transmittance have not been designed to scrutinize spectra measured by the devices themselves. A way of the investigation of the spectra is to sequentially measure both reflectance and transmittance spectra with the same accuracy and to compare the spectra with the law of energy conservation. Thus the accuracy of the measured spectra can be estimated. We have already completed this investigation method for the measurements of regular reflectance and transmittance of specular samples using a STAR GEM ${ }^{\circledR}$ Type 2 (Scatter, Transmission and Absolute Reflection measurements using a Geminated Ellipsoid Mirror) and the method is called a self-diagnosis [4] of measured spectra.

Because the majority of objects around us reflect and transmit light diffusely with a few exceptions like mirrors and windows, the BRDF and BTDF of the scattered light as well as absolute TIR and TIT are important. Our goal is to develop a STAR GEM Type 3 as a scatterometer, which allows us to measure high-precise absolute TIR and TIT based on the spatial distribution of the scattered light measured only by a CCD camera.

\section{STAR GEM TYPE 2 AS A REGULAR REFLECTOMETER}

Until the early 1980s a hemi-ellipsoidal mirror was studied as a regular reflectometer and as a scatterometer [1]. It became clear that a fatal flaw of the ellipsoidal mirror was inter-reflections between a sample and a detector, which were located at two focuses, through the intermediation of 
the ellipsoidal mirror. When the reflectance of a sample became higher, the inter-reflections became larger. For example, Sullivan and Allen [1] estimated the size of the inter-reflection error by employing an averaging sphere at the detector focus. For an actual sample reflectance of 0.70 , inter-reflections would cause the reflectance to be measured as 0.77 . The research and development using a hemiellipsoidal mirror stopped after the 1980s. One reason is the inter-reflection and another is that the reflectance of the inner wall of the integrating sphere increased and its performance was improved.

We have studied a regular reflectometer using two ellipsoidal mirrors, which has been named STAR GEM Type 2, since 2000. The STAR GEM Type 2, which can absolutely measure both regular reflectance and transmittance of specular samples, is composed of two beltshaped ellipsoidal mirrors. The belt-shaped ellipsoidal mirror in Fig.1(a) is fabricated by cutting an ellipsoid of revolution along two planes at the same distance from an equatorial plane and also along a plane perpendicular to a rotation axis through one focus. In the STAR GEM Type 2, two equivalent belt-shaped ellipsoidal mirrors are combined together along the cutting plane (an AB plane in Fig.2(b)) such that three focal points (F0, F1, and F2) are aligned (a CD line in Fig.2(b)) and two equatorial planes correspond to each other. This CD line is a GEM axis. A sample is placed at a common focus (F0) and two rotating mirrors are placed at two remaining focuses (F1, F2) as shown in Fig.2(a). Photographs of the GEM and the STAR GEM Type 2 are shown in Fig.2(b) and 2(c). (a)

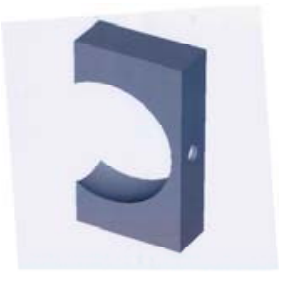

(b)

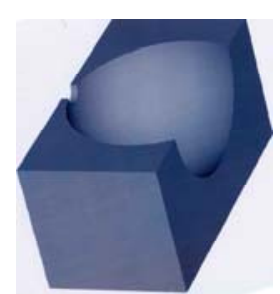

Fig.1. Belt-shaped ellipsoidal mirror (a) and a quarter-ellipsoidal mirror (b), respectively, are the original mirrors used in the described reflectometer and scatterometer.

The STAR GEM Type 2 has many important advantages and high performance. The STAR GEM is designed for specular reflection and specular transmission studies of substrates, films on substrates, coatings and contaminants on surfaces. The STAR GEM can independently make both absolute reflectance and absolute transmittance measurements at the same accuracy based on the symmetry $\mathrm{X}$ method [5] mentioned below. This advantage realizes the scrutinization method of the measured spectra. The STAR GEM can obtain calculated absorptance from the measured reflectance and transmittance. This advantage is helpful in estimating the influence of the inter-reflection inside ellipsoidal mirrors. The STAR GEM comes with a Geminated Ellipsoid Mirror (GEM), which allows the incident angle to be continuously changed from $1^{\circ}$ to $88^{\circ}$. Because the light source and detector are placed outside the
GEM and because the narrow ellipsoidal mirrors such as belt-shaped ellipsoidal mirrors instead of a hemi-ellipsoidal mirror are used, the effect of the inter-reflections, which influences measured reflectance and transmittance, becomes small and negligible. As a result it is realized that the measurement errors calculated from absolute regular reflectance and transmittance are less than $1 \%$.

(a)

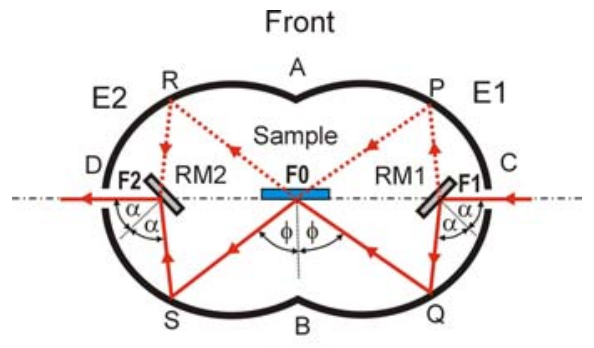

(b)

Back

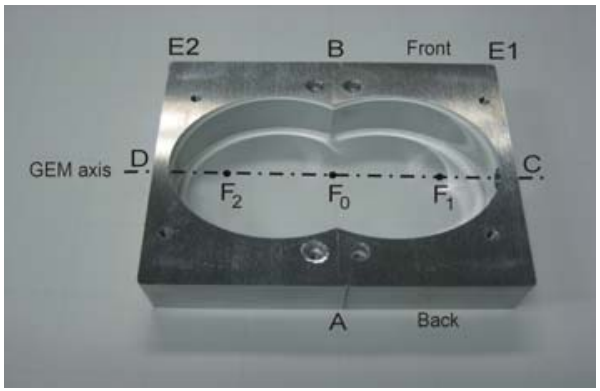

(c)

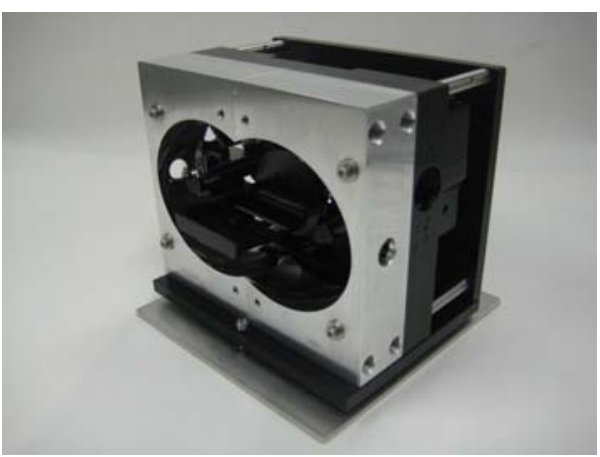

Fig.2. Cross section along the equatorial plane (a), photograph of the GEM (b), and photograph of the STAR GEM Type2 as a regular reflectometer $(\mathrm{c})$.

However, the belt-shaped ellipsoidal mirror (E2) cannot gather all diffuse light scattering from a diffuse sample. Author tried to make the BRDF/BTDF measurements by independently rotating both an E2 mirror around a GEM axis and an RM2 mirror around an F2 in Fig.2(a) and (b) using the STAR GEM Type 2 [6]. This way is similar to the goniometric method and it takes a long time to make the $\mathrm{BRDF} / \mathrm{BTDF}$ measurements. It becomes clear that it is not so easy to get TIR/TIT by integrating the BRDF/BTDF over a portion of the sphere around the sample, too.

\section{StRUCtURE OF STAR GEM TYPE 3 AS A SCATTEROMETER}

In a scatterometer the sample and the optical detection system must be placed at the common focus and third focus, respectively, facing toward the ellipsoidal mirror. This setup 
is the same as that in the previous scatterometer with a hemi-ellipsoidal mirror. In order to reduce the interreflections, two kinds of ellipsoids are prepared - a beltshaped and a quarter ellipsoidal mirror in Fig.1(a) and 1(b). The quarter ellipsoidal mirror is fabricated by cutting an ellipsoid along the equatorial plane and also along a plane perpendicular to the rotation axis through one focus, so that a volume ratio of the quarter to a whole ellipsoid is larger than quarter but a solid angle of the quarter acquired by the sample, which is placed at a common focus (F0), is $\pi$ steradians. Our new scatterometer using the belt-shaped (E1) and the quarter (E2) ellipsoidal mirrors is shown in Fig.3(a) and Fig.3(b), where a common focus (F0), a focus (F1) of the E1 mirror and a focus (F2) of the E2 mirror are aligned. A sample is placed at $\mathrm{F} 0$, a plane rotation mirror (RM1) is placed at F1 and the optical detection system is placed at F2. The first reason why the belt-shaped ellipsoidal mirror is chosen as an incoming ellipsoid is to make the absolute measurements of both reflectance and transmittance according to a modified symmetry X method mentioned below. The second is to reduce the interreflections with decreasing the surface area of the E1 mirror. The third is also to reduce the inter-reflections in the way that the light source is placed outside the GEM. The fourth is the ability to change an incident angle to the sample by the rotation of the RM1 mirror.

The symmetry X method [5] has been invented to measure absolute reflectance of a specular sample. Upper and lower sides of the STAR GEM Type 2 in Fig.2(a) are named Front and Back, respectively. Two reflection measurements can be made in Fig.2(a). One optical path is RM1-Q-Sample-SRM2 (RBB, in brief - the first $\mathrm{R}$ means a reflection measurement, the second $\mathrm{B}$ means a back path before the sample holder, and the last B means a back path after the sample holder) and is shown as a solid red sequential line.

(a)

(b)
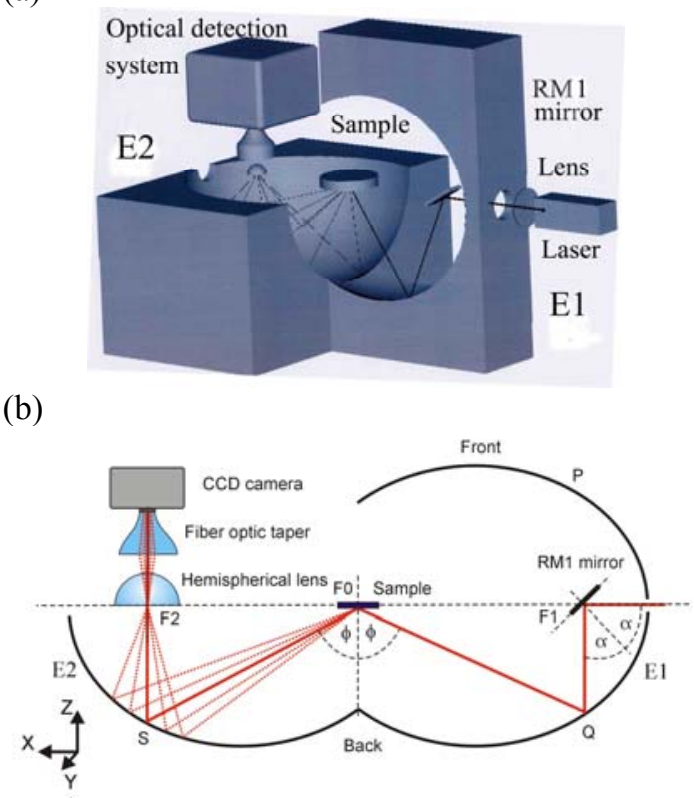

Fig.3. 3D drawing of a STAR GEM type 3 as a whole (a), and cross section along an equatorial plane of the STAR GEM Type3 (b).
The other optical path is RM1-P-Sample-R-RM2 (RFF the second $\mathrm{F}$ means a front path before the sample holder and the last $\mathrm{F}$ means a front path after the sample holder) and is shown as a dotted red sequential line. Two background measurements can be made in Fig.2(a). One path is RM1-Q-F0-R-RM2 (BBF - the first B means a background measurement) and the other is RM1-P-F0-SRM2 (BFB). When the superposition of two optical paths measuring reflection from the sample agrees everywhere with the superposition of two optical paths measuring background without the sample in Fig.2(a), any optical losses at all optical components are completely compensated by the calculation of the geometric means of four signal intensities. Consequently, the absolute regular reflectance of the specular sample can be obtained.

The modified symmetry $\mathrm{X}$ method is explained as follows. Although the rotation mechanics of the E1 and the RM1 mirrors are omitted in Fig.3(a) and 3(b), the E1 mirror and the RM1 mirror can be rotated independently around the axis of the ellipsoid (around $\mathrm{x}$-axis) and around the axis perpendicular to the equatorial plane through the F1 focus (around y-axis), respectively. Upper and lower sides of the scatterometer are named Front and Back, respectively. For the background measurement the RM1 mirror is directed to the upper side and one optical path is a sequential line of RM1-P-F0-S-RM2 (BFB) in Fig.3(b). The other path is a sequential line of RM1-Q-F0-S-RM2 (BBB), when the E1 mirror is rotated by 180 degrees around the $\mathrm{X}$-axis. For the reflection measurement the RM1 mirror is directed to the lower side and the sample is placed at F0 and light reflected from the diffuse sample spreads around $\mathrm{S}$. One optical path is a sequential line of RM1-Q-Sample-S neighborhood-RM2 (RBB). This RBB path is just shown in Fig.3(b). The other path is a sequential line of RM1-P-Sample-S neighborhoodRM2 (RFB), when the E1 mirror is rotated by 180 degrees around the $\mathrm{x}$-axis. If the sample is specular, the superposition of two reflection paths of RBB and RFB agrees everywhere with the superposition of two background paths of BFB and BBB. Then any optical losses at all optical components in Fig.3(a) and (b) are completely compensated by the calculation of the geometric means of four signal intensities. Consequently, the absolute regular reflectance of the specular sample can be obtained. If the sample is diffuse, the superposition of RBB and RFB agrees partially with the superposition of BFB and BBB. Consequently, the absolute reflectance of the diffuse sample may be measured.

Two types of the optical detection system at F2 are adopted. These are composed of a hemispherical lens, a fiber optic taper and a photo-detector, which is either a CCD camera or a photodiode. The first hemispherical lens works to gather all diffuse light from the sample in $\pi$ steradians and to narrow the spread angle of the diffuse light depending on the diameter and refractive index of the hemispherical lens. The study of the hemispherical lens using the ray tracing discloses two points mentioned above and also that the hemispherical lens has a focal curved surface. This discovery surprises us and helps us to get a clear image using a CCD camera. In Fig.4 the diameter and refractive index of the hemispherical lens of S-LAH79 are $10 \mathrm{~mm}$ and 
2.003 , respectively. In an example, a bundle of blue lines on the left of the flat plane of the hemispherical lens in Fig.4 represents parallel rays at an incident angle of 80 degrees. After the bundle passes through the hemispherical lens, the bundle progresses at an outgoing angle of about 30 degrees and its parallel rays converge to a point, which exists at the distance of about two times of the radius of the hemispherical lens. 11 convergent points on the right of the hemispherical lens in Fig.4 agree with 11 bundles at an incident angle of $80,60,45,30,15,0,-15,-30,-45,-60,-80$ degrees. In order to get a clear image, a large aperture of the fiber optic taper is set on the focal curved surface and its small aperture also coincides with an image sensor of the CCD camera in Fig.3(b). The angular resolution is determined by the number of pixels on the image sensor and is about 0.5 degrees ( 0.0003 steradians). The weak point of our detection system using the hemispherical lens is that the image of the perimeter is compressed too much, as a result the angular resolution becomes worse in the perimeter.

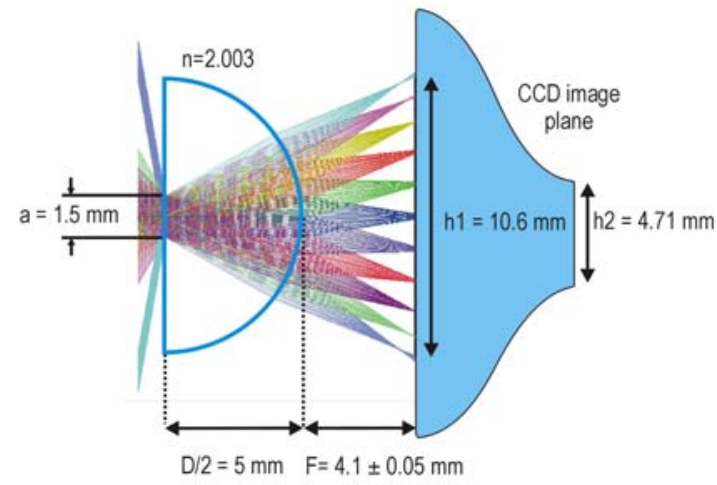

Fig.4. A focal curved surface of a hemispherical lens exists at a distance of about two times of its radius and corresponds to a large aperture of a fiber optic taper.

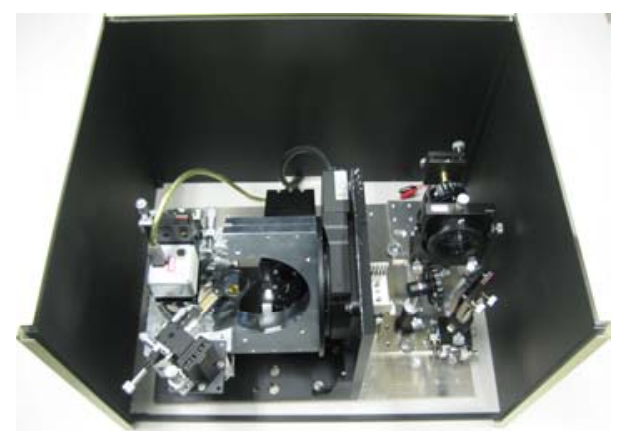

Fig.5. A prototype of a STAR GEM Type 3 as a scatterometer

A prototype of the STAR GEM Type 3 as a scatterometer is shown in Fig.5. The prototype surrounded by an aluminum box is shielded from the room light. Its size is $600 \mathrm{~mm}(\mathrm{~W}) \times 500 \mathrm{~mm}(\mathrm{D}) \times 350 \mathrm{~mm}(\mathrm{H})$. The E1 mirror is a belt-shaped ellipsoidal mirror and is rotatable. The E2 mirror is a quarter ellipsoidal mirror and is fixed. Two rotation stages using a stepping motor are installed for the E1 mirror and the RM1 mirror. The positions of the sample and the optical detection system are independently manually adjusted.
Specialized software has been developed, which simplifies the measurement set-up and allows fully automated control of the measurements.

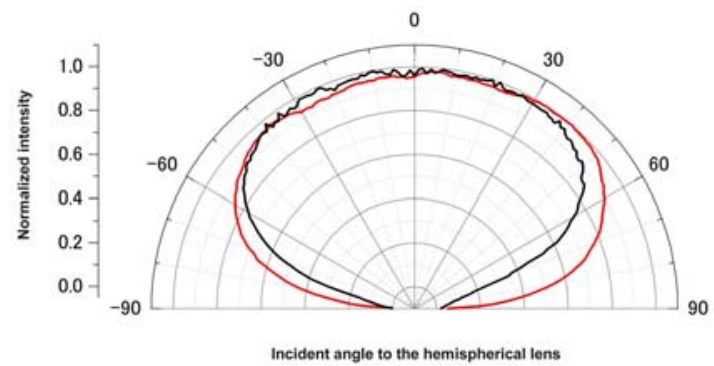

Fig.6. Efficiency of a hemispherical lens. Red and black curves are with and without a hemispherical lens, respectively.

\section{RESUltS AND DISCUSSION}

The light source was a He-Ne laser of $633 \mathrm{~nm}$. The effect of the hemispherical lens was investigated at first. The optical detection system was only a hemispherical lens and a Si photodiode with a large photosensitive area, which was located at the focal curved surface of the hemispherical lens. A beam was scanned along a semicircle of the quarter ellipsoidal mirror by rotating the E1 mirror with a fixed RM1 mirror. In Fig.6, the red curve was obtained with the hemispherical lens and the black curve without it. Using the hemispherical lens the visual angle was larger by 30 degrees.

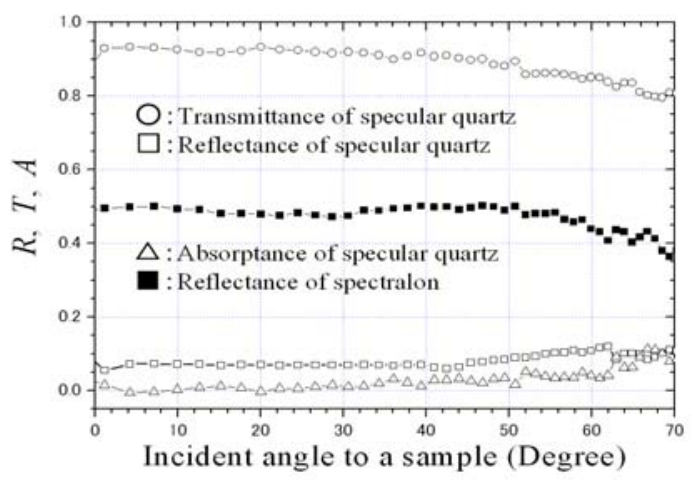

Fig.7. $R, T$ and $A$ of a specular quartz and spectralon

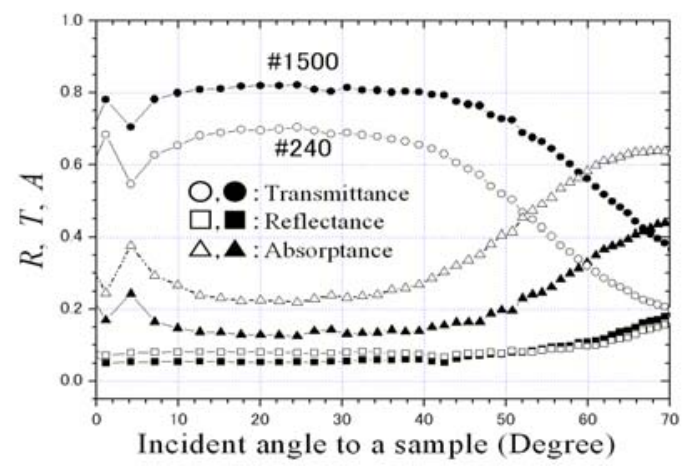

Fig.8. $R, T$ and $A$ of a ground quartz of $\# 240$ and $\# 1500$ 
(a)

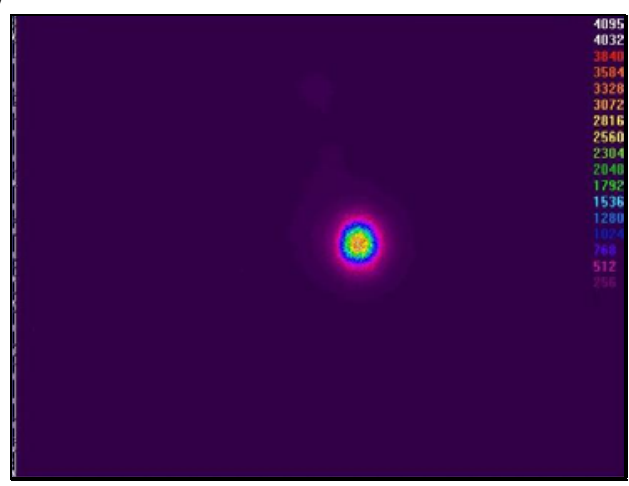

(b)

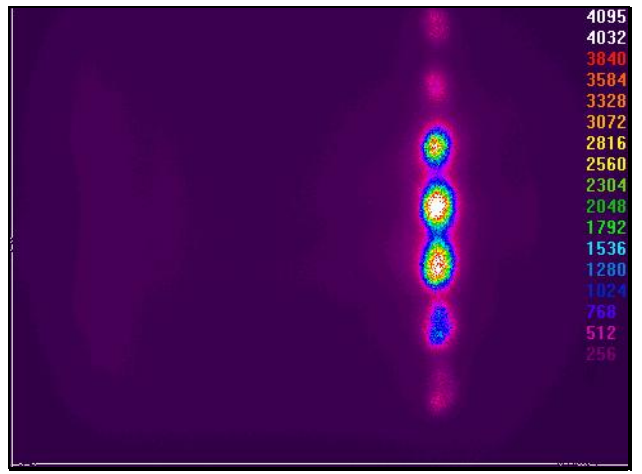

Fig.9. Two photographs captured by the CCD camera for an irradiation laser beam (a) and for light scattering from a diffraction grating (b), respectively.

(a)

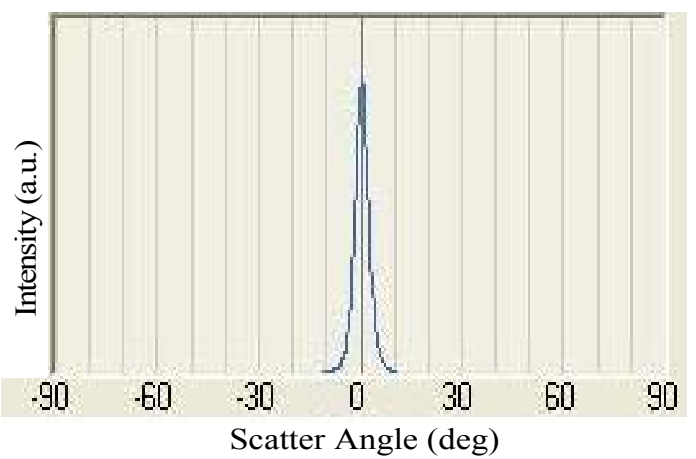

(b)

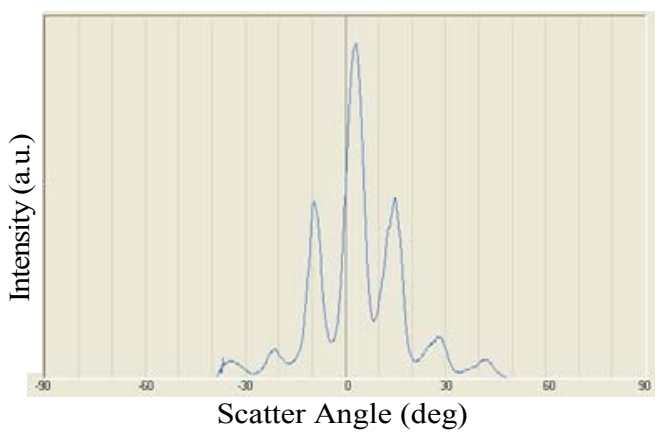

Fig.10. Spatial distributions of an irradiation laser beam (a) and of light scattering from the diffraction grating (b), which are obtained from Fig.9.
The absolute reflectance $(R)$ and transmittance $(T)$ measurements of several samples were made based on the modified symmetry X method. The samples were a specular quartz plate, spectralon as a perfect diffuse material, and two kinds of ground quartz of $\# 240$ and $\# 1,500$ as a partial diffuse material [7]. In Fig.7, open circles, open squares and open triangles are $T, R$ and absorptance $(A=1-R-T)$ of the specular quartz, respectively, and solid squares are $R$ of spectralon. In the range of an incident angle less than 50 degrees, $A$ is almost zero, which is an expected value of the pure quartz. $R$ of spectralon is almost 0.5 at the incident angle less than 50 degrees, which is also an expected value, because the quarter ellipsoidal mirror is reflecting at $\pi$ steradians. In Fig.8, open circles, open squares, and open triangles are $T, R$, and $A$ of the $\# 240$ and solid circles, solid squares, and solid triangles are $T, R$, and $A$ of the $\# 1,500$. In this case $A$ is not zero but about 0.1 , because the spatial distribution of light scattering from the ground samples is not uniform but partially diffuse.

The spatial distributions were measured by the optical detection system using the CCD camera. A photograph of an irradiation laser beam is shown in Fig.9(a) and the other photograph of light scattering from a diffraction grating is shown in Fig.9(b). When Fig.9(a) and (b) are compared, both pictures are clear images. The reason is that the focal curved surface of the hemispherical lens coincides effectively with the image sensor of the CCD camera using the fiber optic taper. Fig.10(a) and (b) are 2D plottings of light intensity distribution along a selected line of images captured by the CCD camera displayed in Fig.9(a) and (b), respectively. The full width at half maximum is about 5 degrees from Fig.10 (a). This value is the spatial resolution of our STAR GEM Type 3. The scatterometer can measure clear diffraction patterns until third order of the diffraction grating in Fig.10(b).

\section{CONCLUSION}

A new scatterometer, which comprises two ellipsoidal mirrors, one rotating mirror, a sample holder, a hemispherical lens, a fiber optic taper and a CCD camera or a photodiode, has been developed. The measurement time required by the scatterometer is around a few minutes and is incomparably shorter than the time required by a goniometric system. The spatial resolution of the scatterometer is about 5 degrees. Absolute total integrated reflectance of specular and perfect diffuse samples and spatial distribution from the diffraction grating were measured successfully.

\section{REFERENCES}

[1] Workman, J., Springsteen, A.W. (1997). Applied Spectroscopy. San Diego: Academic Press.

[2] Shitomi, H., Saito, I. (2009). A new absolute diffuse reflectance measurement in the near-IR region based on the modified double-sphere method. Metrologia, 46, S186-S190.

[3] Apian-Bennewitz, P. (2010). New scanning goniophotometer for extended BRTF measurements. In Proceedings of SPIE, Vol. 7792, 779200-1779200-20. 
[4] Kawate, E. (2008). An optical accessory for absolute reflection and transmission measurements in the wavelength region from $0.24 \mu \mathrm{m}$ to $25 \mu \mathrm{m}$. In Proceedings of SPIE, Vol. 7065, 70650M-1$70650 \mathrm{M}-11$.

[5] Kawate, E. (2003). Symmetry X system and method for absolute measurements of reflectance and transmittance of specular samples. Applied Optics, 42, 5064-5072.
[6] Kawate, E. (2009). Diffuse reflectance and transmittance measurements using a STAR GEM optical accessory. In Measurement 2009: $7^{\text {th }}$ International Conference on Measurement, 20-23 May 2009. Bratislava, Slovakia: Institute of Measurement Science SAS, 270-273.

[7] Lukianowicz, Cz., Karpinski, T. (2003). Scatterometry of ground surfaces. Measurement Science Review, 3 (3), 21-24.

Received December 20, 2011. Accepted April 16, 2012. 